\title{
Proposta metodológica para quantificação de acilaçúcares em folíolos de tomateiro
}

\author{
Gabriel M Maciel'; ${ }^{1}$ Ernani C Silva ${ }^{2}$ \\ ${ }^{1}$ UFU-ICIAG, LAGEN, R. Goias 2000, Vila Nova, 38500-000 Monte Carmelo-MG; gabriel.maciel@iciag.ufu.br; ${ }^{2}$ UFSJ, C. Postal 56, \\ 35701-970 Sete Lagoas-MG; clarete@ufsj.edu.br
}

\begin{abstract}
RESUMO
Um dos grandes problemas enfrentados na tomaticultura é o ataque constante de pragas. A partir do uso de espécies selvagens de tomateiro, tornou-se possível a obtenção de genótipos com resistência a pragas. Tal resistência tem sido associada à presença de aleloquímicos denominados acilaçúcares, que são ésteres de ácidos graxos. Para a quantificação dos teores de acilaçúcares utiliza-se o método colorimétrico permitindo assim a seleção indireta de genótipos com alto teor deste aleloquímico. Este método vem sendo utilizado predominantemente nos programas de melhoramento genético do tomateiro visando resistência a pragas. Apesar da grande utilização do método colorimétrico na seleção indireta de genótipos de tomateiro com resistência a pragas, algumas etapas deste método demandam bastante tempo dos laboratoristas que a executam. Devido à importância do método colorimétrico e na tentativa de melhorar a eficiência e padronização de leitura, este trabalho foi realizado com o objetivo de avaliar novos métodos para quantificação dos teores de acilaçúcares presentes nos folíolos de tomateiro. Dentre os métodos propostos, quando se utilizou o espectrofotômetro Multiskan FC com filtro de $620 \mathrm{~nm}$ e doses de $100 \mu \mathrm{L}$ por poço ( 96 poços), foram obtidos resultados similares aos do método padrão. Esta proposta metodológica permitiu maior eficiência nas análises, repetibilidade, maior padronização dos resultados, diminuindo a chance de erros durante a execução, além da análise simultânea de maior número de amostras. Conclui-se que o referido método é viável e eficiente, podendo ser utilizado na quantificação de acilaçúcares em folíolos de tomateiro visando seleção indireta de genótipos resistentes a pragas.
\end{abstract}

Palavras-chave: Solanum pennellii, método colorimétrico, pragas, seleção indireta.

\author{
ABSTRACT \\ Methodological proposal to quantify acylsugars in tomato \\ leaflets
}

One of the major problems in tomato production is the frequent attack of pests. The use of wild tomato species made it possible to obtain pest resistant genotypes. Such resistance has been linked to the presence of allelochemicals called acylsugars, which are esters of fatty acids. The colorimetric method is normally used to quantify the levels of acylsugars, allowing an indirect selection of genotypes with high content of allelochemicals. This methodology has been used predominantly in the breeding programs of tomato to obtain pestresistant genotypes. Despite the wide use of the colorimetric method for indirect selection of tomato genotypes with pest resistance, some steps of this methodology take great demand of time. Knowing the importance of the colorimetric method and, attempting to improve the efficiency and standardization of reading, we tried to evaluate new methodologies for quantifying the levels of acylsugars present in tomato leaflets. Among the proposed methods, when using the spectrophotometer Multiskan FC with filter of $620 \mathrm{~nm}$ and doses of $100 \mu \mathrm{L}$ per well (96 wells), we obtained results which were similar to the standard methodology. This methodological approach allowed for greater efficiency in the analysis, repeatability, greater standardization of results reducing the chance of errors during execution, besides the simultaneous analysis of many samples. Concluding, this method is feasible and effective and can be used to quantify acylsugars in tomato leaflets with the purpose of indirect selection of resistant genotypes to pests.

Keywords: Solanum pennellii, colorimetric method, pests, indirect selection.

\section{(Recebido para publicação em 26 de agosto de 2013; aceito em 4 de abril de 2014) (Received on August 26, 2013; accepted on April 4, 2014)}

$\mathrm{E}$ mbora seja uma das mais importantes plantas cultivadas, o tomateiro é acometido de graves problemas fitossanitários (Suinaga et al., 2003). Os problemas fitossanitários foram agravados pela expansão da área de cultivo, o que favoreceu o surgimento de pragas e doenças de difícil controle, que afetam significativamente a sua produção (Filgueira, 2000).

A partir de espécies selvagens de tomateiro, tornou-se possível a obtenção de genótipos com resistência a um grande número de pragas. Tal resistência tem sido associada à presença de aleloquímicos denominados de acilaçúcares (AA), que são ésteres de ácidos graxos. Estes aleloquímicos podem atuar impedindo a ovoposição, a alimentação ou, ainda, exercendo efeito deletério no desenvolvimento de determinadas fases de um artrópode-praga (Resende et al., 2002; Resende et al., 2006; Resende et al., 2008; Maciel et al., 2011). Gonçalves et al. (2007) indicaram que altos teores de AA se devem à ação de um alelo recessivo, com dominância incompleta no sentido de baixos teores.

Atualmente, a quantificação dos teores de AA vem sendo realizada através da utilização do método colorimétrico, conforme descrito por
Resende et al. (2002). Utilizando-se desse método, a seleção indireta de genótipos com resistência a pragas em tomateiro mediada por AA derivados de S. pennellii foi extensivamente utilizada (Hawthorne et al., 1992; Rodriguez et al., 1993; Juvik et al., 1994; Liedl et al., 1995; Resende et al., 2002; Gonçalves et al., 2006; Resende et al., 2006; Saeidi et al., 2007; Maciel et al., 2009; Silva et al., 2009; Gonçalves Neto et al., 2010; Maciel et al., 2011).

Maluf et al. (2010) e Gonçalves Neto et al. (2010) demonstraram que linhagens avançadas de tomateiro com alto teor de AA apresentaram altos níveis de 
resistência à Tuta absoluta. Em virtude da dominância incompleta, foi possível a obtenção de híbridos resistentes a artrópodos-praga, mesmo entre linhagens com alto teor e linhagens com baixo teor de AA(Maluf et al., 2010; Maciel et al., 2011). Avaliando diferentes combinações híbridas entre linhagens com altos teores e linhagens com baixos teores de AA, foi comprovado amplo espectro de resistência à mosca-branca (Bemisia tabaci biótipo B), à traça-do-tomateiro (Tuta absoluta) e ao ácaro-rajado (Tetranychus urticae) (Maluf et al., 2010). A eficiência do método colorimétrico (Resende et al., 2002) também foi comprovada quanto à seleção indireta de genótipos resistentes a $B$. tabaci biótipo $\mathrm{B}$, à traça-do-tomateiro e ao ácaro rajado (Maluf et al., 2010).

Apesar da grande utilização do método colorimétrico na seleção indireta de genótipos de tomateiro com resistência a pragas, algumas etapas do método proposto por Resende et al. (2002) apresentam considerável demanda de tempo de execução pelos laboratoristas. Devido à importância do método colorimétrico na seleção indireta e na tentativa de melhorar a eficiência e padronização de leitura, este trabalho foi realizado com o objetivo de avaliar novos métodos para quantificação dos teores de AA presentes nos folíolos de tomateiro, buscando maior eficiência de execução e repetibilidade.

\section{MATERIAL E MÉTODOS}

O experimento foi instalado em casa de vegetação, de 15 de março a 28 de julho de 2012, na HortiAgro Sementes em Ijaci-MG (21 ${ }^{\circ} 14^{\prime} 16^{\prime \prime} \mathrm{S} ; 4^{\circ} 08^{\prime} 00^{\prime \prime} \mathrm{O}$; $918 \mathrm{~m}$ de altitude).

A quantificação dos teores de acilaçúcares (AA) das amostras foi realizada na UFLA, utilizando o método padrão de Resende et. al. (2002) que, após a extração dos AA, realiza leituras pelo espectrofotômetro UV-160 1 PC Visible Spectrophotometer da marca Shimadzu, o qual possui a capacidade de realizar uma amostra por leitura, a partir de uma cubeta com aproximadamente $2 \mathrm{~mL}$ de solução.

O novo método proposto foi execu- tado no Centro de Indexação de Vírus da UFLA, utilizando a mesma solução obtida pelo método de Resende et al. (2002) após extração dos AA, sendo, porém, a leitura realizada pelo espectrofotômetro Thermo Scientific (MultiskanFC) e o software Skan IT 2.5.1, o qual realiza leituras em placas de poliestireno de fundo chato (marca COSTAR 3590), com capacidade de leitura imediata de 96 poços (amostras).

Os genótipos foram semeados em 15 de março de 2012, em bandejas de poliestireno expandido com substrato comercial à base de fibra de coco. Decorridos 30 dias após a semeadura, as mudas de cada genótipo foram transplantadas para vasos de 5 litros contendo o mesmo substrato comercial utilizado na formação das mudas e mantidos em casa de vegetação. Os genótipos avaliados no experimento foram: acesso selvagem LA-716 (S. pennellii, com alto teor de acilaçúcares), TOM-684 (linhagem pré-comercial com baixo teor de acilaçúcares), TOM-687 (linhagem pré-comercial com alto teor de acilaçúcares obtida a partir do cruzamento interespecífico entre $S$. pennellii x $S$. lycopersicum), TOM-689 (linhagem pré-comercial com alto teor de acilaçúcares obtida a partir do cruzamento interespecífico entre $S$. pennellii x $S$. lycopersicum) e híbrido comercial 'Bravo' (testemunha comercial com baixo teor de acilaçúcares) (Maluf et al., 2010; Maciel et al., 2011).

Decorridos 38 dias do transplantio, no início da fase de florescimento, realizou-se a coleta dos discos nos folíolos para quantificação dos teores de AA, seguindo método padrão proposto por Resende et al. (2002).

Foram retirados seis discos foliares por genótipo, em oito repetições, com auxílio de um vazador (cano metálico de $1 \mathrm{~cm}$ de diâmetro), os quais foram acondicionados em tubos de ensaio previamente identificados e levados imediatamente para o Laboratório de Química Orgânica da UFLA. Em cada tubo foi adicionado $1 \mathrm{~mL}$ de diclorometano e agitados por 30 segundos em aparelho vórtex, visando promover a extração do aleloquímico. Posteriormente foram retirados os folíolos e evaporado o solvente e, em seguida, adicionou-se $0,5 \mathrm{~mL}$ de $\mathrm{NaOH} 0,1 \mathrm{~N}$, dissolvido em metanol, evaporando-o em seguida. O resíduo foi mantido em alta temperatura $\left(100^{\circ} \mathrm{C}\right)$, no qual foi adicionado $4 \mathrm{~g} \mathrm{~L}^{-1}$ de metanol por três vezes, em intervalos de 2 minutos. Após a evaporação total do metanol, o resíduo foi dissolvido em 0,4 mL de água. Para a inversão da sacarose, foi adicionado $0,1 \mathrm{~mL}$ de $\mathrm{HCl}$ $0,04 \mathrm{~N}$, aquecendo-se por 5 minutos até a ebulição. Decorrido esse tempo, a solução obtida foi resfriada e, em seguida, adicionou-se $0,5 \mathrm{~mL}$ do reagente $\mathrm{A}+$ reagente $B$ (proporção de 25:1) (Nelson, 1960) que foi aquecido em ebulição por 10 minutos. Em seguida, os tubos contendo as amostras foram resfriados em água corrente, adicionando-se 0,5 $\mathrm{mL}$ de arseniomolibdato e agitando por 15 segundos, em aparelho vórtex. Logo em seguida, as amostras foram submetidas à leitura espectrofotométrica, para absorbância na faixa de 540 nm. Após extração foram feitas leituras pelo método descrito por Resende et. al. (2002) utilizando uma cubeta com aproximadamente $2 \mathrm{~mL}$ de solução.

Após extração de AA das amostras originais, foram testadas nove propostas metodológicas de leitura (Tabela 1) utilizando-se o espectrofotômetro Thermo Scientific (MultiskanFC) e o software Skan IT 2.5.1 e comparadas com a leitura proposta por Resende et. al. (2002), na qual se utiliza o espectrofotômetro UV-160 1 PC Visible Spectrophotometer da marca Shimadzu. Para tanto, foram utilizadas as mesmas soluções obtidas, após extração dos AA, referentes às oito repetições de cada genótipo utilizadas para leitura pelo método padrão de Resende et al. (2002). Portanto, essas soluções foram pipetadas em placa de poliestireno de fundo chato (marca COSTAR 3590), com capacidade de leitura imediata de 96 poços (amostras), utilizando-se uma pipeta regulada nas doses de 10,50 e $100 \mu \mathrm{L}$. Respectivas doses foram utilizadas para todos os genótipos em estudo (LA-716, TOM-684, TOM-687, TOM-689 e 'Bravo' F1), sendo previamente conhecidos os teores de AA de cada genótipo quantificados pela metodologia de Resende et. al. (2002). Em seguida foram feitas leituras com filtros de 405, 450, 620 $\mathrm{nm}$ referentes a cada dose pipetada na placa $(10,50$ e $100 \mu \mathrm{L})$ totalizando nove 
propostas metodológicas. Utilizou-se o delineamento em blocos casualizados, sendo as médias comparadas entre si utilizando o teste de Tukey, a 5\% de probabilidade por meio do programa estatístico SISVAR (Ferreira, 2000). Avaliou-se ainda a variância $\left(\mathrm{S}^{2}\right)$ ocorrida em cada genótipo em relação às oito repetições tanto no teste colorimétrico (Resende et al., 2002) quanto nas nove propostas metodológicas, com o intuito de verificar qual método fornece leituras com maior repetibilidade.

\section{RESULTADOS E DISCUSSÃO}

O método colorimétrico proposto por Resende et al. (2002) foi eficiente na quantificação dos teores de acilaçúcares (AA) (Tabela 1). Estes resultados confirmaram a eficiência do referido método de acordo com estudos realizados por diversos autores que a utilizaram na quantificação de AA em folíolos de tomateiro (Gonçalves et al., 2006; Resende et al., 2006; Saeidi et al., 2007; Maciel et al., 2009; Maluf et al., 2010; Gonçalves Neto et al., 2010; Maciel et al., 2011). Embora o método colorimétrico (Resende et al., 2002) seja amplamente utilizado, uma das maiores dificuldades encontradas é a grande demanda de tempo na execução pelos laboratoristas.

Entre as nove novas propostas metodológicas analisadas, apenas uma (Filtro $620 \mathrm{~nm}$ e dose $100 \mu \mathrm{L}$ ) apresentou leituras similares ao método colorimétrico padrão descrito por Resende et al. (2002), não diferindo estatisticamente (Tabela 1). As variações ocorridas entre o método padrão de Resende et al. (2002) e a melhor metodologia (Filtro $620 \mathrm{~nm}$ e Dose $100 \mu \mathrm{L}$ ) entre os mesmos genótipos foram de apenas: 1,84; 0,85; 0,$13 ; 0,04 ; 1,05$ nanomols.cm² de área foliar $^{-1}$ nos genótipos LA-716, TOM684, TOM-687, TOM-689 e Bravo F1, respectivamente. Estas diferenças, nas concentrações de AA obtidas nos dois métodos não foram consideradas significativas. Os outros métodos de leitura não permitiram resultados próximos do método padrão de Resende et al. (2002), não sendo recomendados para identificar genótipos resistentes a pragas via seleção indireta.

Outra dificuldade encontrada pelo método colorimétrico (Resende et al., 2002) está relacionada com a variação da solução final obtida para leitura. A quantidade de solução (dose) utilizada na cubeta do espectrofotômetro UV-160 1 PC Visible Spectrophotometer da marca Shimadzu pode influenciar a leitura e consequentemente atribuir valores incorretos no teor de AA presente nos folíolos de tomateiro.

Durante as leituras realizadas no espectrofotômetro UV-160 1PC Visible Spectrophotometer da marca Shimadzu, além das leituras serem feitas individualmente, o que resulta em maior tempo de execução nas análises, outra etapa que contribui na interferência das leituras é a necessidade de limpar a cubeta, durante o intervalo entre a leitura de cada amostra, uma vez que resíduos de soluções anteriores podem interferir na leitura da próxima amostra analisada. Utilizando o espectrofotômetro MultiskanFC com filtro de $620 \mathrm{~nm}$ e dose de $100 \mu \mathrm{L}$ por poço, estas interferências foram eliminadas, uma vez que são realizadas 96 leituras imediatas, sob placas de poliestireno (marca COSTAR 3590) descartáveis. Além de eliminar a interferência na leitura, quando comparado com o método de Resende et al. (2002), ressalta-se ainda a maior eficiência na execução por não ser necessário limpar toda vez a cubeta no intervalo entre cada leitura. Por outro lado, observou-se também maior padronização do método em que se utilizou filtro de $620 \mathrm{~nm}$ e dose de $100 \mu \mathrm{L}$ por poço (Tabela 1 ). Os valores obtidos de variância $\left(\mathrm{S}^{2}\right)$ que ocorreram entre as oito amostras do mesmo genóti-

Tabela 1. Nove propostas metodológicas para quantificação dos teores de acilaçúcares (nanomols) em folíolos de tomateiro comparadas com o método padrão (Resende et al., 2002) e variância (S²) ocorrida em cada método entre as amostras [nine methodological approaches to quantify the levels of acylsugar (nanomols) in tomato leaflets compared with the standard methodology (Resende et al., 2002) and variance $\left(\mathrm{S}^{2}\right)$ occurred in each methodology between samples). Lavras, UFLA, 2012.

\begin{tabular}{|c|c|c|c|c|c|c|c|c|c|c|}
\hline \multirow{2}{*}{ Método } & \multicolumn{10}{|c|}{ Genótipo } \\
\hline & LA-716* & $\mathbf{S}^{2 * *}$ & TOM-684 & $S^{2}$ & TOM-687 & $\mathrm{S}^{2}$ & TOM-689 & $\mathbf{S}^{2}$ & Bravo F1 & $\mathbf{S}^{2}$ \\
\hline Filtro $405 \mathrm{~nm}(10 \mu \mathrm{L})$ & $25,54 \mathrm{c}$ & 9,77 & $7,78 \mathrm{~d}$ & 2,92 & $21,78 \mathrm{~b}$ & 0,27 & $20,92 \mathrm{~b}$ & 6,07 & $7,30 \mathrm{~d}$ & 2,31 \\
\hline Filtro $405 \mathrm{~nm}(50 \mu \mathrm{L})$ & $22,96 \mathrm{c}$ & 2,11 & $15,69 \mathrm{~b}$ & 0,69 & $17,25 \mathrm{c}$ & 2,14 & $18,12 \mathrm{bc}$ & 2,13 & $17,69 \mathrm{~b}$ & 0,49 \\
\hline Filtro $405 \mathrm{~nm}(100 \mu \mathrm{L})$ & $57,31 \mathrm{a}$ & 27,70 & $36,43 \mathrm{a}$ & 14,27 & $46,68 \mathrm{a}$ & 32,37 & $46,40 \mathrm{a}$ & 3,67 & $41,38 \mathrm{a}$ & 1,01 \\
\hline Filtro $450 \mathrm{~nm}(10 \mu \mathrm{L})$ & $11,30 \mathrm{~d}$ & 5,36 & 3,84 efg & 1,24 & $8,07 \mathrm{~d}$ & 0,07 & $7,92 \mathrm{e}$ & 3,19 & $3,54 \mathrm{f}$ & 1,08 \\
\hline Filtro $450 \mathrm{~nm}(50 \mu \mathrm{L})$ & $8,19 \mathrm{~d}$ & 0,72 & $5,39 \mathrm{def}$ & 0,05 & $6,84 \mathrm{~d}$ & 0,32 & $6,66 \mathrm{e}$ & 0,21 & $5,93 \mathrm{ef}$ & 4,33 \\
\hline Filtro $450 \mathrm{~nm}(100 \mu \mathrm{L})$ & $22,07 \mathrm{c}$ & 5,03 & $11,68 \mathrm{c}$ & 12,50 & $16,74 \mathrm{c}$ & 1,47 & $14,38 \mathrm{~d}$ & 3,88 & $11,55 \mathrm{c}$ & 1,40 \\
\hline Filtro $620 \mathrm{~nm}(10 \mu \mathrm{L})$ & $10,26 \mathrm{~d}$ & 0,51 & $2,69 \mathrm{~g}$ & 0,30 & $9,83 \mathrm{~d}$ & 0,21 & $6,62 \mathrm{e}$ & 2,07 & $2,44 \mathrm{f}$ & 0,23 \\
\hline Filtro $620 \mathrm{~nm}(50 \mu \mathrm{L})$ & $9,61 \mathrm{~d}$ & 0,48 & $3,55 \mathrm{fg}$ & 0,01 & $8,11 \mathrm{~d}$ & 0,37 & $6,73 \mathrm{e}$ & 0,28 & $3,59 \mathrm{f}$ & 0,05 \\
\hline Filtro $620 \mathrm{~nm}(100 \mu \mathrm{L})$ & $38,43 \mathrm{~b}$ & 5,57 & $6,43 \mathrm{de}$ & 1,28 & $22,25 \mathrm{~b}$ & 0,88 & $15,24 \mathrm{~cd}$ & 6,70 & $6,52 \mathrm{ef}$ & 0,55 \\
\hline $\begin{array}{l}\text { Padrão (Resende } \text { et al., } \\
\text { 2002) }\end{array}$ & $36,59 \mathrm{~b}$ & 10,70 & $7,28 \mathrm{~d}$ & 1,80 & $22,38 \mathrm{~b}$ & 2,23 & $15,20 \mathrm{~cd}$ & 5,70 & $5,47 \mathrm{e}$ & 1,80 \\
\hline
\end{tabular}

10,76

16,31

11,16

11,63

8,98

*Médias seguidas da mesma letra não diferem entre si nas colunas pelo teste de Tukey a 5\% de probabilidade (means followed by the same letter do not differ in columns by Tukey test at $5 \%$ probability) $* * \mathrm{~S}^{2}=$ variância observada nas oito repetições em cada tratamento (variance observed in eight replicates for each treatment). 
po foram, em praticamente todos os genótipos avaliados e em termo absoluto, menores que o método padrão proposto por Resende et al. (2002).

Os menores valores de variância obtidos com o uso do espectrofotômetro MultiskanFC com filtro de $620 \mathrm{~nm}$ e doses de $100 \mu \mathrm{L}$ por poço (96 poços) proporcionaram maior eficiência nas análises e maior padronização dos resultados. Conclui-se que o referido método é viável e eficiente, podendo ser usado na quantificação de AA em folíolos de tomateiro visando seleção indireta de genótipos resistentes a pragas.

\section{AGRADECIMENTOS}

À FAPEMIG, ao CNPq, à CAPES pelo auxílio financeiro e concessão de bolsas; à UFLA, ao Centro de Indexação de Vírus de Minas Gerais e à empresa HortiAgro Sementes Ltda e ao Departamento de Química da UFLA.

\section{REFERÊNCIAS}

FERREIRA DF. 2000. Análises estatísticas por meio do Sisvar para Windows 4.0. In: REUNIÃO ANUAL DA REGIÃO BRASILEIRA DA SOCIEDADE INTERNACIONAL DE BIOMETRIA, 45. Anais... São Carlos: UFSCAR. p. 255-258.

FILGUEIRA FAR. 2000. Novo manual de olericultura. Viçosa: UFV. 402 p.

GONÇALVES NETO AC; SILVA VF; MALUF WR; MACIEL GM; NIZIO DAC; GOMES LAA; AZEVEDO SM. 2010. Resistência à traça-do-tomateiro em plantas com altos teores de acilaçúcares nas folhas. Horticultura
Brasileira 28: 203-208.

GONCALVES LD; MALUF WR; CARDOSO MG; GOMES LAA; NASCIMENTO IR. 2007. Herança de acilaçúcares em genótipos de tomateiro provenientes de cruzamento interespecífico. Pesquisa Agropecuária Brasileira 42: 699-705.

GONÇALVES LD; MALUF WR; CARDOSO MG; RESENDE JTV; CASTRO EM; SANTOS NM; NASCIMENTO IR; FARIA MV. 2006. Relação entre zingibereno, tricomas foliares e repelência de tomateiros a Tetranychus evansi. Pesquisa Agropecuária Brasileira 41: 267-273.

HAWTHORNE DJ; SHAPIRO JA; TINGEY WM; MUTSCHLER MA. 1992. Trichomeborne and artificially applied acylsugars of wild tomato deter feeding and ovoposition of the leaf miner Liriomyza trifolii. Entomologia Experimentalis et Applicata 65: 65-73.

JUVIK JA; SHAPIRO JA; YOUNG TE; MUTSCHLER MA. 1994. Acylglucose from wild tomato alters behavior and reduce growth and survival of Helicoverpa zea and Spodoptera exigua (Lepidoptera: Noctuidae). Journal of Economic Entomology 87: 482-492.

LIEDL BE; LAWSON DM; WHITE KK; SHAPIRO JA; COHEN DE; CARSON WG; TRUMBLE JT; MUTSCHLER MA. 1995. Acylsugars of wild tomato Lycopersicon pennellii alters settling and reduces oviposition of Bemisia argentifolii (Homoptera: Aleyrodidae). Journal of Economic Entomology 88: 742-748.

MACIEL GM; MALUF WR; SILVA VF; GONÇALVES NETO AC; GOMES LAA. 2011. Híbridos pré-comerciais resistentes a Tuta absoluta obtidos de linhagem de tomateiro rica em acilaçúcares. Horticultura Brasileira 29: 151-156.

MACIEL GM; MALUF WR; SILVA VF; GONÇALVES NETO AC; HAYATA L; CARVALHO RC; MORETTO P; LICURSI V; MORETTO DP. 2009. Heterose e capacidade combinatória em linhagens de tomateiro na obtenção de híbridos com teores intermediários de acilaçúcares. Horticultura Brasileira 27: 1161-1167.
MALUF WR; MACIEL GM; GOMES LAA; CARDOSO MG; GONÇALVES LD; SILVA EC; KNAPP M. 2010. Broad-spectrum arthropod resistance in hybrids between high and low-acylsugar tomato lines. Crop Science 50: 439-450.

NELSON NA. 1960. Photometric adaptation of Somogyi method for determination of glucose. Journal of Biologic Chemistry 153:375-380.

RESENDE JTV; MALUF WR; CARDOSO MG; FARIA MV; GONCALVES LD; NASCIMENTO IR. 2008. Resistance of tomato genotypes with high level of acylsugars to Tetranychus evansi. Scientia Agricola 65: 31-35.

RESENDE JTV; MALUF WR; FARIA MV; PFANN AZ; NASCIMENTO IR. 2006. Acylsugars in tomato leaf lets confer resistance to the South American tomato pinworm, Tuta absoluta. Scientia Agricola 63: 20-25.

RESENDE JTV; CARDOSO MG; MALUF WR; SANTOS CD; GONÇALVES LD; RESENDE LV; NAVES FO. 2002. Método colorimétrico para quantificação de acilaçúcar em genótipos de tomateiro. Ciência e Agrotecnologia 26: 1204-1208.

RODRIGUEZAE; TINGEY WM; MUTSCHLER MA. 1993. Acylsugars of Lycopersicon pennelli deter settling and feeding of the green peach aphid (Homoptera: Aphididae). Journal of Economic Entomology 86: 34-49.

SAEIDI Z; MALLIK B; KULKARNI RS. 2007. Inheritance of glandular trichomes and two-spotted spider mite resistance in cross Lycopersicon esculentum 'Nandi' and $L$. pennellii 'LA2963'. Euphytica 154: 231-238.

SILVA VF; MALUF WR; CARDOSO MG; GONÇALVES NETO AC; MACIEL GM; NÍZIO DAC; SILVA VAS. 2009. Resistência mediada por aleloquímicos de genótipos de tomateiro à mosca-branca e ao ácaro-rajado. Pesquisa Agropecuária Brasileira 44: 12621269.

SUINAGA FA; CASALI VWD; SILVA DJH; PICANÇO MC. 2003. Dissimilaridade genética de fontes de resistência de Lycopersicon spp. a Tuta absoluta (Lepidoptera: Gelechiidae). Revista Brasileira de Agrociência 9: 371-376. 